\title{
PLM Interoperability with Information Systems
}

\author{
Aurélie Bissay and Kamal Cheballah \\ GreenLight-IT, Private Research Center in Informatics, Lyon, France \\ \{abissay, kcheballah\} @greenlight-it.fr
}

\begin{abstract}
Industrial companies evolve in a highly competitive economic environment in which economic constraints require permanent adjustment. Whatever the size of the company, it has to manage and optimize in continuous way business processes in order to innovate with respect to the environment. These requirements make information systems at the heart of this issue as far as these systems manage and adjust information flows from the perspectives of business processes. Our work focuses on improving the modeling of information systems.
\end{abstract}

Keywords: Information system, business process, Interoperability, PLM.

\section{Introduction}

Information systems can play a key role in the success of a company. Indeed, business leaders are usually faced to a number of crucial decisions (resources allocation, choice of an economic model, etc.) which lead the company in the long term to achieve a sustainable advantage. These choices can only be made from the data available to the company; information has become an even greater value that it contributes to achieve the goals of the company.

Information systems provide the valuable information that the company needs for efficient operations, effective management and therefore to obtain or keep its competitive advantage. A good usage of the information system and its adaptation to the strategic objectives of the company helps to thrive in a highly competitive economy.

The implementation of an information system within a structure is a costly investment both in terms of human resources and budget.

Large companies deal mostly with writing specifications and perform afterwards specific developments, rigid and expensive.

Taking into consideration their organization, more often rigid, specific aspects of their needs must be addressed, which have additional costs to deal with. However, in the context of SMEs / SMIs, such an approach is difficult to handle. This kind of structures needs to perceive returns on investment in a short term and has no or little means to develop the specific needs. Besides, in order to manage the diversity of customers and / or contractors they have to face the changing environment.

Our research works are keenly interested in the structure type of SME / SMIs. The approach discussed in this paper aims to bring these structures flexibility in their information systems without the need for specific developments. 
The first part of this paper focuses on the study of the state of the art in terms of related fields in line with our research objectives. In fact, our work deals primarily with information systems as well as interoperability between the information systems components. We discuss afterwards business processes aspect since they form the basis of the company's activities and they are performed in the information system through workflows.

Finally, we propose a framework called 'INTERPRO' that aims to shorten the interoperability within different systems. This paper is concluded by highlighting the need of an active approach dealing with the characterization of usages in order to enhance the help provided to user while modeling their system.

\section{Information Systems and Business Processes}

\subsection{Information System and Related Components}

An information system (IS) is an organized set of resources (hardware, software, data and procedures) enabling grouping, classifying, processing and dissemination of information within a specific environment [1].

The information system is the communication vehicle in an organization. Its structure consists of numerous resources (people, hardware, software) organized in order to: collect, store, process and communicate information.

The information system is built upon a set of business processes and related interactions, and not only databases or software.

Nowadays, most of information systems use computer equipment, software, and new information and communication technologies to transfer resources into data and various information products in a secure way when it comes to sensitive data.

Information systems include databases, enterprise resource planning system (ERP), customer relationship management system (CRM), computer-aided production the management tools, business applications, data and applications servers, storage systems and security systems in order to ensure data integrity and persistence.

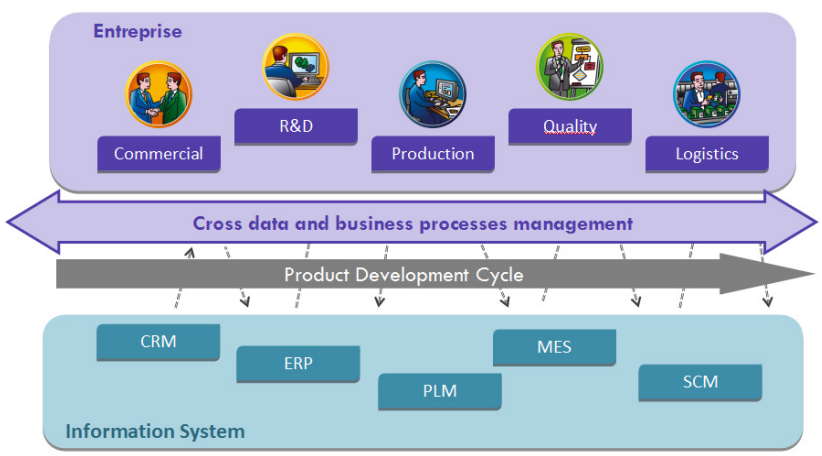

Fig. 1. The components of the information system in companies 
The information system includes usually several components. The schema shown below illustrates the numerous software that can be part of the information system.

The schema above illustrates the components of an information system namely:

- Customer Relationship Management - CRM, dedicated to customer relationship management.

- Enterprise Resource Planning - ERP, dealing with the management of operational processes, integrating all functions such as the human resource management, accounting and financial management, as well as sale, distribution, supply and so forth.

- Product Lifecycle Management - PLM, consisting of capitalizing all information related to an industrial product.

- Manufacturing Execution Systems - MES, covering the main aras of production control.

- Supply Chain Management - SCM, aiming to improve and automate supplying by reducing inventories and delivery times.

Besides the variety of the aforementioned tools and systems, one can highlight the diversity of data models, human machine interfaces, business processing and technology environments that comprise an information system. This diversity leads to an additional complexity from the perspectives of integration and interoperability.

In addition, among these different systems, two main categories of tools may be considered apart:

- Systems that deal with the capabilities of a company, cost and time.

- Systems that deal product knowledge.

The first category deals mainly with resources (such as equipments, human capabilities, subcontractors, customers, financial capacity ...) managed through computer services and operational services and departments of a company; whereas the other category deals with technological skills (research, development, designers, strategic quality...).

\subsection{Interoperability and Integration of Systems}

Entirely partitioned, the two aforementioned categories of information systems operate in a completely independent manner. The common aspects are very few, and are limited in general to coding a specific request and sending a product structure of a system to another. However, the optimization of the overall functions of a company leads necessary to:

- release and increase the number of exchanges points,

- $\quad$ enable users interacting with the two categories of systems.

System integration aims to assemble different parts of a system in order to ensure compatibility and relevant functioning of the whole system. In other words, system 
integration it meant to "break-down" functional and organizational barriers within companies so that the "whole" can be seen as coherent and harmonious. However, works from this area have shown that organizational adaptation of companies has to go first through an adaptation of technological tools and applications. Since the late ninety, the paradigm of interoperability has taken precedence over the concept of integration as being more focused on technological aspects which are intensely evolving [2].

Indeed, system interoperability is a way, among others, to facilitate integration [3]. Interoperability can be defined as the ability to communicate with remote systems in order to access to and use their functionalities [4], whereas the integration is a much broader concept. According to Chen [5], this mutation from integration to interoperability is not a matter of technical change; it reflects, above all, the changing economic, organizational and social aspects of society regarding the globalization of industry and manufacturing.

Within information systems, some components are often integrated so that they can communicate among each other. Generally speaking, this kind of integration is the most structured and is achieved through the establishment of common exchange models, interfaces, languages or syntax. For instance, we can highlight the case of MES and ERP interoperability that aims to enhance and accelerate the flows between the schedules made within the ERP and the process control performed within the MES. (Cf. Figure 2).

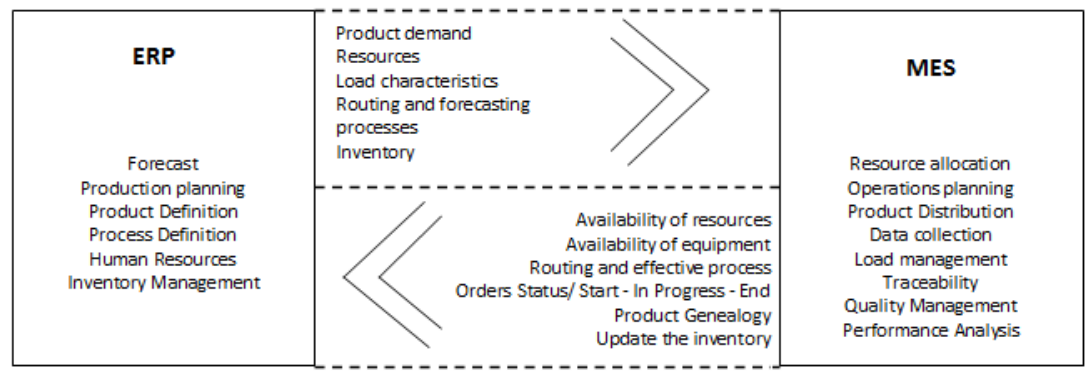

Fig. 2. Sample of data exchanged at the level of ERP and MES systems [2]

However, this approach to make two software tools interoperable is restrictive and does not take into account the totality of the information system and related components.

\subsection{Business Processes}

Business process management is one of the major concerns of companies. The constant need for optimizing performances in order to remain competitive in their market is one of the most important drivers. A high-quality and efficient modeling lead to facilitate communication between actors and mainly to streamline business units. Processes are an actively concerned concept in many areas. In the industrial areas, and 
according to Davenport [6], this concept is defined as "scheduling activities across time and place, having a start and an end, with clearly defined inputs." According to Vernadat [7], "the process is a set of steps partially ordered and performed to satisfy requirements of a company". This definition highlights the notion of objective related to an industrial purpose. We can also quote the definition of El Mhamdi [8] which defines the process as "a combination of activities, mobilizing multiple skills, taking place in time and being motivated by an objective."

On the whole, "a process is a set of activities, undertaken within a defined purpose. The responsibility of performing all or a part of the activities by an actor is defined as a role. The way how the process uses resources may be conditioned by internal or external events. The arrangement of activities corresponds to the structure of the process" [9]. This definition is particularly relevant since it highlights the main components of the process: purpose, activities, roles, resources an event.

Modeling business processes aims to represent its structure and functioning according to a specific point of view and with a certain level of detail in order to improve performances.

Modeling may provide an answer to different objectives:

- The establishment of a quality approach namely to achieve a certification quality, may lead to represent the functioning of the company as a set of processes.

- From a "computational" perspective, process models form a basis for software setting.

\section{An Approach for Modeling an Agile Information System}

This research work is conducted within the specific context of SMEs / SMIs and aims to provide a flexible information system through a specific layer.

\subsection{The Global Approach}

The different components of an information system (PLM, ERP, CRM...) include a workflow integrated engine. Each process is therefore performed through the system according to its activity. In other words, an ordering process will be handled within the ERP; a product design process will be managed within the PLM, and so forth.

However the boundary between each of these components of the information system is somehow indistinguishable. Indeed, each component may overlap with other components.

For instance, a product nomenclature can be found in both the ERP and the PLM systems. These cases are numerous and lead to ask about the tool which will be performing these processes at the boundary between two or more components.

In addition, for the users of these systems, this boundary is not clearly defined. This may lead to some resistance as far as their interactions become more complex. They have to learn to cope with and handle the different components according to their needs. 
In order to make easier data manipulation, our research work aims to define a process driven layer in the upstream of the system.

The following figure provides a synoptic view of the global information system. Each user connects to the proposed layer to perform the tasks and actions he is responsible for via HMI that depending upon the relevant needs will query the components of the system (ERP or PLM ...).

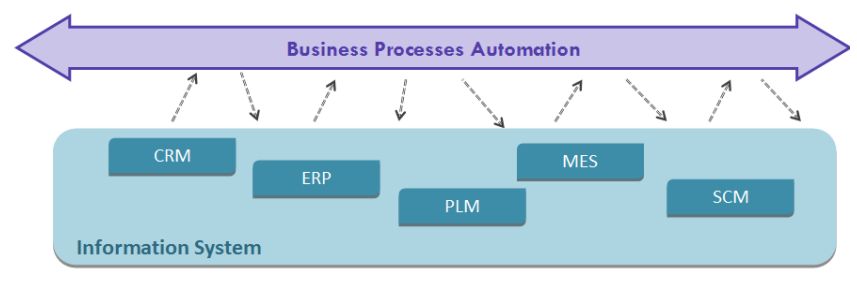

Fig. 3. Proposal for modeling an information system

This layer is meant to be as generic as possible in order to not generate costly custom specific developments during the deployment. On the one hand, this layer aims to enables automated business processes implementation. On the other hand, it enables interactions with the different components of the system through a set of Human Machine Interfaces (HMI) dedicated to the components.

The objective of this research is twofold:

- To enable a smooth interoperability between information system components and provide a clear vision of the information system for users. This first objective takes into account change management issues.

- To work on the generation of HMI under constraints related to the environment but also to the used equipments.

However, some difficulties may rise from this work mainly from the perspectives of architecture and modeling aspects of this application. Therefore, it is necessary to consider a meta-model as generic as possible in order to predict the most possible cases.

\subsection{The Proposed Framework INTERPRO}

Our research work aims to provide a framework, we called INTERPRO, described in the following schema: 


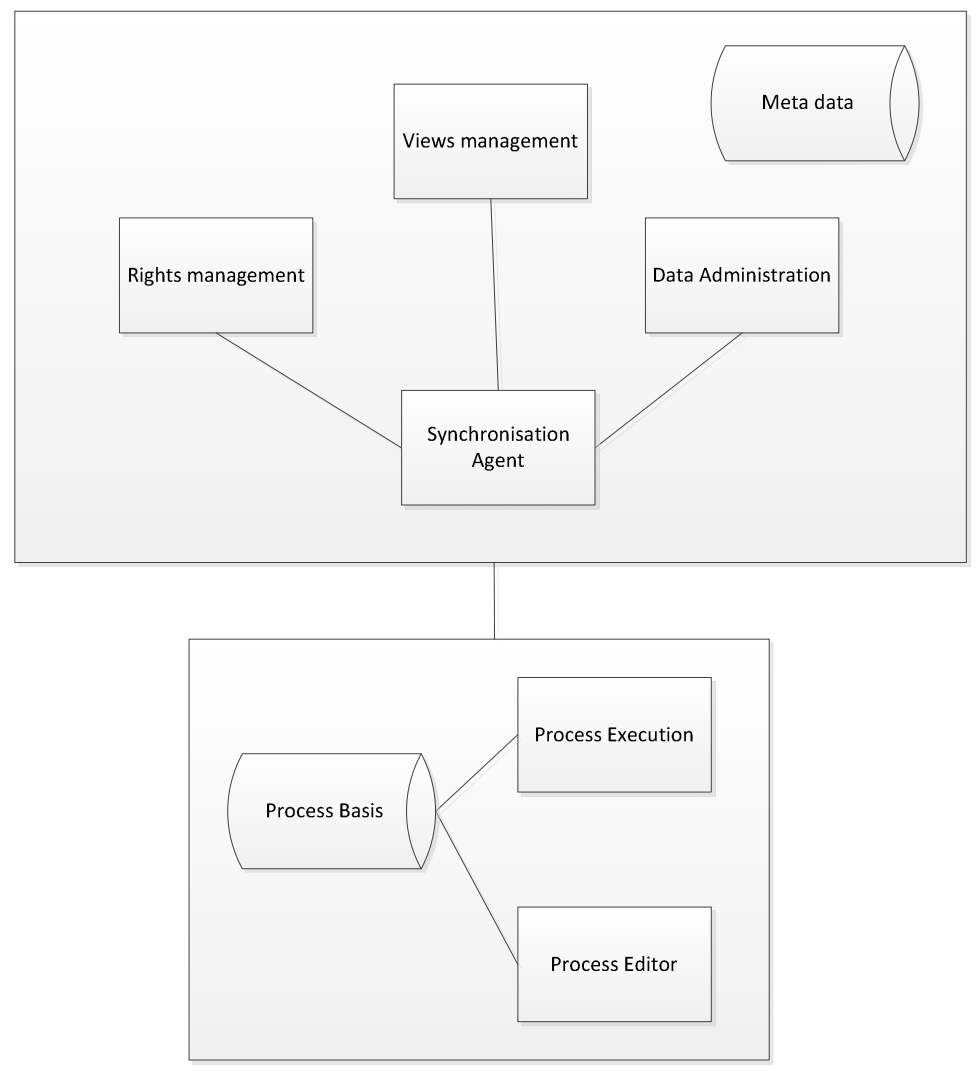

Fig. 4. The proposed framework INTERPRO

The framework consists of a system organized in two levels:

- the database level dealing with rights, data and views management, and

- the orchestration level dealing with treatments and processes management to ensure the function expected by an organization.

On one hand, within the first level, a synchronization agent aims to synchronize the effects of evolutions on rights, views and data management; these effects are an intrinsic phenomenon occurring in a company and not often taken into consideration. On the other hand, within the second level, business processes are modeled and performed. The first level acts dynamically on the process editor and the process execution components according to the evolutions occurring within the information system.

Within the data base, the administration system enables modeling and describing:

- Simple data (integer, reals, pointers, thesaurus, lists, dictionaries ...)

- External data

- direct access (data sources, ...),

- Non-direct access (access through services, specific treatments,). 
Relations between data are modeled using a semantic network. Data is enriched enabling therefore the definition of triggers as per events occurring on data and as follows:

- Before and after insertion of an object

- Before and after updating an attribute

- Before and after the evolution of an object

- ...

The usage of finite state trees and a dependency tree enables the synchronization agent performing its role, even though impacts are generally realized within scripts performed by the triggers.

The views management system enables the description of all areas or set of areas related to communications with users in order to handle the data described above. Views are:

- simple (direct access to data),

- complex (access on a graph of relations),

- black box (access to a step of an external process of third party system).

The rights management system enables the description of the organization of users, their rights and obligations regarding data.

Besides, at the second level, the process editor enables the definition of one or more processes and carries out the arrangement of steps, generally connected by various types of transitions. Whereas the process execution enables the orchestration of the process execution by implementing all related parameters. It provides the orchestration of external process in order to implement the business process.

Also, in the same way we introduce a data description language, we propose a process description language.

With this in mind, a process is defined as a collection of ordered steps;

Process ::=[Step $]^{*}$

Step $\mathrm{i}_{\mathrm{i}}:=$ Sub Process $\left(\right.$ system $_{\mathrm{i}}$,exchange_context_area) | Hybride_Configuration $\mathrm{i}_{\mathrm{i}}$

Hybride_Configuration ${ }_{i}::=$ [ Hybride interactive Assembly | Interative Third party I Non interactive Third party ]+

The hybrid interactive is a user exchange system enabling checking and updating information coming from different information systems aiming to collaborate together.

The hybrid interactive is our proposal in order to represent a step within a business process, considered hence as the collaborative point between $\mathrm{n}$ processes. These $\mathrm{n}$ processes, working together at a specific point with the interactive hybrid, may be either synchronous or asynchronous. The same approach is applied for data sources with indirect access to data that can be synchronous or asynchronous. 


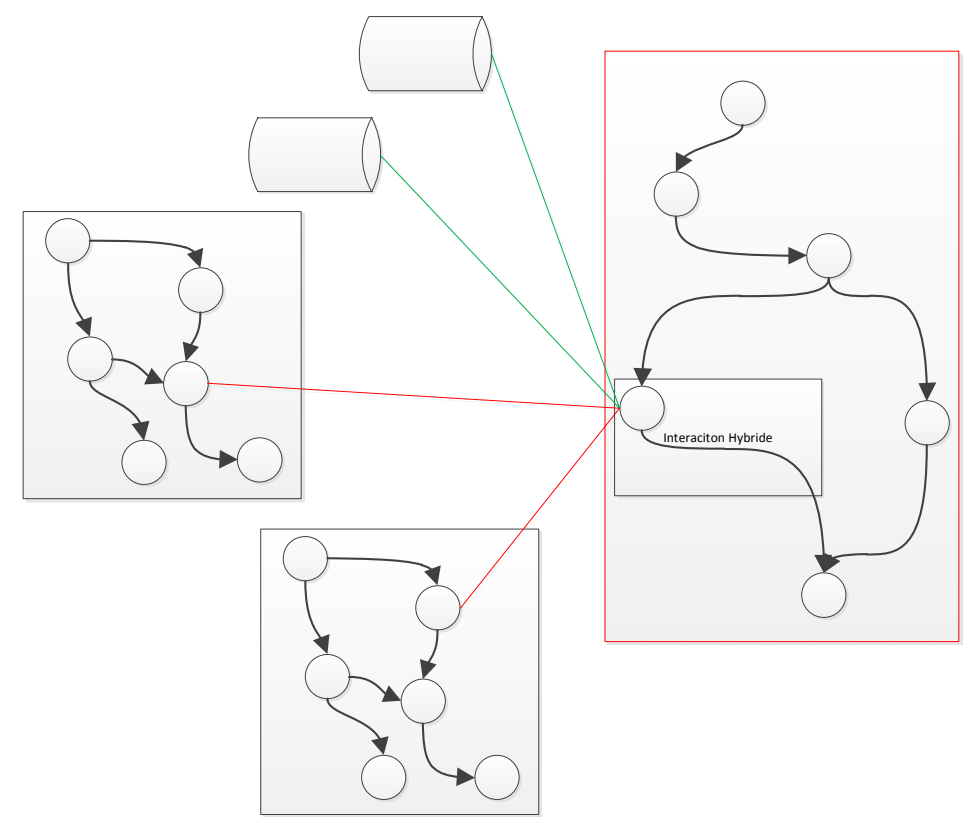

Fig. 5. Steps from external processes

In the schema above, we highlight a hybrid interactive step that calls a set of steps from external processes and various data sources. With this in mind, one of the difficult aspects that we may face is to enable the extraction of some of the contextual information from sub-processes and bring it back to the hybrid interactive level.

Indirect access to datas are defined by the following mode :

- for the the synchronous mode by :

- the data source description

- the access mode (read, update, delete)

- the technology (sql, web services, ...) and the protocol access for each mode

- the way the errors will be handled

- for asynchronous mode by:

- the data source description

- the access mode (read, update, delete)

- the technology (sql, web services ...) and the protocol access for each mode

- the way the errors will be handled

- the exchange event

- the description of time out mangement

Definition of system sub process

- Interactive mode

- synchronous mode by: 
- container model

- call mode (exe, http, https, request, ...)

- modeling the activation of sub process steps,

- the data source description

- the method to write and read on the context exchange area

- access mode (read, update, delete)

- Description of information exchange

- asynchronous mode:

- not supported in our project

- Non-interactive mode

- synchronous mode by:

- call mode (exe, http, https, request, ...)

- modeling the activation of sub process steps,

- the data source description

- the method to write and read on the context exchange area

- access mode (read, update, delete)

- Description of information to exchange

- asynchronous mode by:

- call mode (exe, http, https, request, ...)

- modeling the activation of sub process steps,

- the data source description

- the method to write and read on the context exchange area

- access mode (read, update, delete)

- Description of information to exchange

- exchange event

- the description of time out management

The INTERPRO Framework and his language description will be prototyped on an existing PLM system as well as an ERP that support some workflow modelisation

\section{Conclusion}

Our proposal is particularly interested in SMEs / SMIs. Their capacity to develop products which enables them to improve their productivity remains limited. But to survive they must adapt as soon as possible to changes in its market.

For this, we propose a framework that enables it to develop without programming knowledge, and adapt its productivity tools only by taking into account its business expertise.

Through this research project we will make a contribution to improving the modeling of information systems. 


\section{References}

[1] De Courcy, R.: Les systèmes d'information en réadaptation, Québec, Réseau international CIDIH et facteurs environnementaux, vol. 1-2(5), pp. 7-10 (1992)

[2] Baïna, S.: Interopérabilité dirigée par les modèles, Doctorat de l'Université Henri Poincaré, Nancy I, France (2006)

[3] Panetto, H., Baïna, S., Morel, G.: Mapping the IEC 62264 models onto the Zachman framework for analysing products information traceability: a case study. Journal of Intelligent Manufacturing (2006) ISSN: 0956-5515

[4] Vernadat, F.B.: Enterprise modelling and integration: principles and applications. Chapman \& Hall (1996) ISBN: 978-0-412-60550-5

[5] Chen, D.: Modélisation d'entreprise pour l'intégration et l'interopéra-bilité des systèmes industriels, Habilitation à diriger des Recherches à l'Université de Bordeaux 1, France (2005, 2006)

[6] Davenport, T.H.: Process innovation. Harvard Business School Press (1993)

[7] Verschoor, A.H., Reijnders, L.: The use of lifecycle mathods by seven major companies. Journal of Cleaner Production 375-382 (1999)

[8] El Mhamedi, A., Lerch, C., Marier, S., Sonntag, M.: Modélisation des activités et des processus des systèmes de production: une approche interdisciplinaire. RAIRO-APII-JESA: Journal Européen des Systèmes Automatisés (31) (1997)

[9] Morley, C., Hugues, J., Leblanc, B., Hugues, O.: Processus métiers et sys-tèmes d'informations: évaluation, modélisation, mise en œuvre, Dunod (2005) 